\title{
New approaches in thermal plasma technology*
}

\author{
Joachim Heberlein \\ High Temperature and Plasma Laboratory, Department of Mechanical Engineering, \\ University of Minnesota, Minneapolis, MN 55455, USA
}

\begin{abstract}
Thermal plasmas offer unique advantages for materials processing, such as high fluxes of heat and of reactant species. Recent developments have concentrated on improving control of these fluxes across the boundaries surrounding the thermal plasma. Secondary discharges (hybrid plasma generators) and pulse modulation of the plasma have been some of the approaches for this end. The use of such methods is described for selected applications. Plasma characterization through advanced models and diagnostics are concentrating on description of plasma instabilities and various nonequilibrium conditions. Understanding of these effects will allow their use for enhanced processing methods.
\end{abstract}

\section{INTRODUCTION}

The major challenges for thermal plasma science and technology have changed over the years. The special characteristics of thermal plasmas were seen to offer an almost unlimited potential as a processing medium, and efforts were focused on reaching an understanding of the fundamental processes and on making use of this largely unknown processing medium in industrial applications. Lack of success in any one application has been associated with a lack of knowledge of the plasma process and the need for more research. Today, the limitations of thermal plasmas as a processing medium have become clearer, and one even encounters occasionally the view that no scientific discoveries can be made any more in thermal plasma technology, and the challenges are now the application of our scientific knowledge to the solution of engineering problems.

Is it true that all the fundamental phenomena in thermal plasma science have been discovered and described? Are there still discoveries to be made which will lead to new applications, or are there materials processing needs which can only be answered by using thermal plasmas, or at least significantly better than with any other processing medium?

In this contribution, I will attempt to provide some answers to these questions by looking at the special characteristics of thermal plasmas as a processing medium, and then discuss the advantages and challenges encountered when thermal plasmas are used in specific applications.

\section{THERMAL PLASMA CHARACTERISTICS}

The special characteristics of thermal plasmas offer a combination of unique advantages:

- Thermal plasmas are a processing medium with one of the highest energy densities; the results are high processing rates, high fluxes of radical species, the potential for smaller installations, a wide choice of reactants, and high quench rates.

- Use of electricity as the primary energy source assures independence from the oxygen potential of the medium and, therefore, a controlled environment. Furthermore, gas flow rates are reduced, control of energy flow is facilitated, and a short response time is possible.

\footnotetext{
${ }^{*}$ Lecture presented at the $15^{\text {th }}$ International Symposium on Plasma Chemistry, Orléans, France, 9-13 July 2001. Other presentations are presented in this issue, pp. 317-492.
} 
The same characteristics that offer these advantages pose special challenges:

- The high energy density leads to strong gradients and frequently to nonuniformities of the process. Also, instabilities are encountered reducing the process yield, and the large number of independent process parameters can make process control difficult.

- Electricity is one of the most expensive forms of energy, and the economics of the process and the installations are frequently unfavorable.

It is clear that the major attraction of using thermal plasmas compared to other processing media is the improved process economics offered by the high rates and compact installations, and any specific process development needs to maintain this advantage.

The disadvantages of thermal plasma processing are not important when the process results in a high value added to the product, because higher process costs and lower yields may be acceptable. However, the trend in thermal plasma processing has been to expand into applications of lower valueadded products, and control of the process and improving reliability by controlling instabilities have become the primary issues.

Figure 1 provides a look at different thermal plasma processing applications in a systematic way by classifying the applications according to the phase of the medium to be processed. It is clear that one can divide the applications into those which make use of the plasma solely as an efficient energy transfer medium, and those where the plasma-generated species are directly involved in the process chemistry. A further division can be made according to the characteristic processing time. Processes with relatively long characteristic times ( $>1 \mathrm{~s}$ ) are less influenced by instabilities of the plasma generation process and of the large-scale turbulence in the mixing region, whereas processing with characteristic process times of $10 \mathrm{~ms}$ or less may be affected by these instabilities.

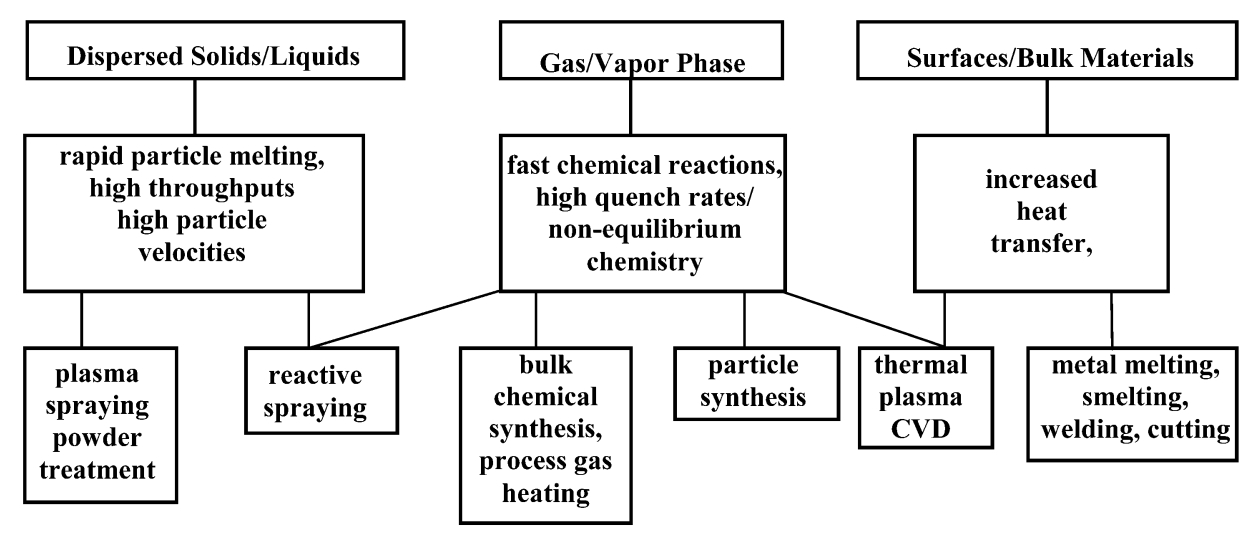

Fig. 1 Overview of thermal plasma processing applications.

Looking at the applications where the plasma is used as an effective source of process heat, we have several plasma-related effects which increase the heat transfer. In the case where the medium to be processed is used as an electrode (transferred arc configuration), these effects are predominantly due to the terms associated with the current transport

$$
q_{A}=j_{e} \phi_{w}+\left(2 \frac{\kappa}{e}+\frac{\phi}{\sigma}\right) j_{e} T_{e}-K_{a} \frac{d T_{a}}{d z}-K_{e} \frac{d T_{e}}{d z}+j_{i}\left(X_{i}-\phi_{w}\right)
$$

The first two terms on the right-hand side represent electron condensation, enthalpy, and thermodiffusion, while the last term associated with the ion flux describes the contribution of the recombination. The remaining terms are the heavy-particle and electron-conduction terms. 
In case of only convective heat transfer, e.g., to a particle, the plasma provides enhanced heat transfer due to the reactive contribution in the thermal conductivity and the Prandtl number

$$
q_{p}=h\left(T_{\infty}-T_{p}\right) ; \quad h=\frac{K}{d_{p}}\left(2.0+0.6 \operatorname{Re}^{\frac{1}{2}} \operatorname{Pr}^{\frac{1}{3}}\right)
$$

$K, K_{\mathrm{a}}, K_{\mathrm{e}}$ - thermal conductivity: total, heavy particles, electrons; $\mathrm{Re}, \mathrm{Pr}-\mathrm{Reynolds}$ number, Prandtl number; $T_{\infty}, T_{\mathrm{a}}, T_{\mathrm{e}}, T_{\mathrm{p}}$-temperature of plasma, atoms and ions, of electrons, particle surface; $j_{e}, j_{i}$-electron and ion current density; $X_{\mathrm{i}}$-ionization potential; $\phi_{\mathrm{w}}$-work function; $\kappa$-Boltzmann constant; $e-$ electron charge; $\sigma$-electrical conductivity; $\phi$-thermal diffusion coefficient.

In the situation where the plasma takes part in the process chemistry, we have to consider that a thermal plasma is a very efficient source of reactive species, i.e., dissociated or ionized radicals. For example, a small plasma torch used for atmospheric pressure plasma spraying operating at $30 \mathrm{~kW}$ with a thermal efficiency of $66 \%$, with a gas flow rate of 0.03 to $0.07 \mathrm{~mol} / \mathrm{s}$ of $\mathrm{Ar}, \mathrm{H}_{2}$ or a mixture of these gases, will provide a fully dissociated plasma flow which may be at an average temperature of between 9000 and $13000 \mathrm{~K}$ (depending on the amount of hydrogen), and up to $30 \%$ ionized. This translates into a flow rate of electrons in the 8-mm-diameter jet at the torch exit of about 5 to $10 \times 10^{21}$ per second. Similar numbers hold for other radicals. To make efficient use of these reactants requires the control of the recombination kinetics between the plasma generator and the medium to be processed. Since the medium to be processed (e.g., a cold gas or a solid or molten surface) is usually at a much lower temperature, delaying recombination requires imposition of nonequilibrium conditions.

\section{SPECIFIC THERMAL PLASMA-PROCESSING APPLICATIONS}

In metal melting and remelting, the plasma is used primarily as an effective source of process heat, making use of the anode heat transfer characteristics of an arc between a cathode and the metal. The relatively long characteristic process times (from $0.1 \mathrm{~s}$ to $\mathrm{min}$ ) reduce the importance of instability effects. With the increased use of recycled scrap material (in 2000, $35 \%$ of the steel was produced from scrap [1]), the metal melting and remelting application has seen considerable expansion. New approaches have concentrated mainly on more efficient overall operation of the installation and less on the control of the plasma heat transfer.

In plasma cutting and welding, the use of a plasma is more economical than using a laser or an electron beam which may provide higher power flux densities. New approaches are driven by improvement of the product quality and process reliability. Examples are the expanded use of pulsing the weld current and of sensors for feedback control in automated welding. Current pulsing and use of signal analysis techniques for interpreting weldpool images or cutting torch voltage traces will further improve process reliability.

In plasma and in wire arc spraying, the plasma provides high heat and momentum transfer rates to melt and accelerate spray particles or to melt the wire tips and atomize the molten metal. Characteristic process times are determined by the particle exposure to the plasma which is in the order of 0.1 to $10 \mathrm{~ms}$, making it susceptible to plasma instabilities. This application has been expanding with the expanded use of coatings required by the increasing demand on structural materials. More and more materials require corrosion or wear-resistant or thermal-barrier coatings. Expansion of the technology into large-scale manufacturing has driven developments for improved economics, process reliability, and control. Process economics is most strongly influenced by the use of lower-cost powders, requiring development of equipment and processes that provide quality coatings with inexpensive powders. Improving reliability requires control of plasma instabilities, and process controls must have sensors with time resolution in the order of $100 \mu \mathrm{s}$. 
Two recent DC torch developments have addressed the issues of arc stability and use of a wider range of powders. In one of these torches, the arc is formed between three cathodes and a single anode, and the powder is injected between the three cathodes into the arc (central injection) rather than into the plasma jet downstream of the anode making the process less dependent on the powder quality [2]. The other torch also uses three cathodes and one anode, however, the plasma gas flow is controlled such that the arcs remain separate [3]. Furthermore, the anode consists of only a ring with a relatively small axial extent, thus fixing the arc length and reducing the plasma instabilities. Other approaches have modified conventional spray torches with the use of gaseous shrouds for fluid dynamic control of the instabilities [4].

Radio-frequency plasma spraying, although in use for many years, has recently seen expanded use. The use of nozzles to increase the plasma jet and spray particle velocity [5] has added to this technique some of the advantages of DC plasma spraying while preserving the central injection and long residence time advantages of the RF process. In particular, the new development of suspension plasma spraying, where a suspension of the deposition precursors is injected in atomized form into the RF plasma, allows the use of a larger variety of deposition precursors [6]. Plasma spraying with a "hybrid" plasma torch employing a DC plasma torch issuing a jet into a RF plasma torch has been described already in 1983 [7]. This technology combines some of the advantages of DC and RF plasma spraying, and only the complexity of the dual power supplies and controls have impeded the wider spread use of this technology.

Numerous new approaches have concentrated on the development of diagnostics for feedback control. Examples are the measurement of the temperatures and velocities of a number of individual spray particles in flight or of an ensemble of spray particles, and adjustment of the process variables to set these particle properties to the desired values [8-10]. Other approaches have concentrated on the development of inexpensive sensors for the measurement of arc and plasma jet characteristics, such as frequency of the emitted sound or of the voltage fluctuations [11] to devise feedback control methods and improve process reliability.

In thermal plasma $C V D$ and the following thermal plasma applications, the plasma is predominantly used as source of specific chemical radicals driving the formation of the desired product.

The advantage of using a thermal plasma are the high fluxes of the deposition precursors resulting in high deposition rates, and the large variety of reactants which can be used. Characteristic process times are the transit times of the chemical species from the injection point to the substrate, and they range from 0.01 to $1 \mathrm{~ms}$. The high deposition rates of high quality films have found commercial use in the deposition of diamond coatings for a variety of applications. Besides the process economy, reliability as provided by the control of the chemical boundary layer between the substrate and the plasma, and the film-substrate interface are the major issues.

A number of approaches have been pursued to increase control of the boundary-layer chemistry while preserving the high precursor fluxes. The goal is to obtain in the boundary layer in front of the substrate a species concentration profile which is independent from the temperature profile. Use of a supersonic plasma jet carrying the deposition precursors into a low-pressure environment is one of the earliest methods to be used for this application $[12,13]$. However, a similar effect has been recently achieved with a lower velocity jet at atmospheric pressure using a secondary glow discharge superimposed onto the DC plasma jet [14]. The secondary discharge between the torch anode serving as the cathode and the substrate as the glow discharge anode increased in a diamond deposition process the electron densities in front of the substrate sufficiently to yield increased atomic hydrogen densities, which in turn resulted in dramatically increased diamond deposition rates. In a similar process, however, in a low-pressure environment, pulsing of the glow discharge between the torch anode and the substrate could affect not only the deposition rate but also the film characteristics (see Fig. 2) [15]. A positive substrate bias led to the deposition of an amorphous or nanocrystalline boron carbide film at deposition rates strongly increasing with substrate bias, while a negative bias resulted in highly stressed crystalline films, with the rate only slowly increasing with increasing bias. Spectroscopic analysis of the 


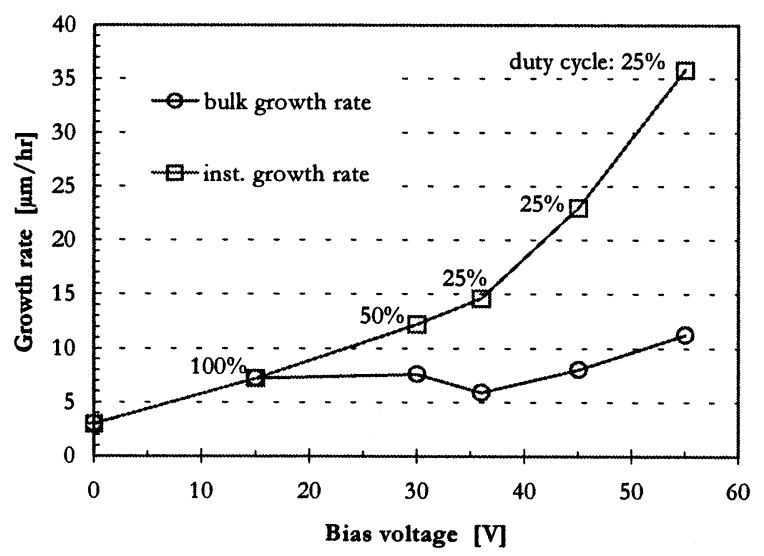

Fig. 2 Increase of the growth rate of a boron carbide film as a function of the substrate bias voltage. The pulsed bias voltage generates a secondary glow discharge superimposed onto the DC arcjet [15].

plasma jet revealed that the bias did not increase the temperatures of the molecules and atoms, but increased the degree of dissociation (probably due to higher electron temperatures), and, therefore, the density of the dominant deposition precursor boron.

Thermal plasma surface treatment other than deposition can be accomplished by using a thermal plasma to generate at high rates reactive species, and use sophisticated fluid dynamic designs to keep their concentrations high while they are transported to the substrate. Characteristic process times are in the millisecond range. An example of such a process is the thinning of a silicon wafer through etching with fluorine atoms or the sterilization of food containers. In one specific application, the reactive species generated in the arc plasma are maintained in a stratified flow arrangement while the plasma is cooling thus allowing surface reactions at low temperatures [16]. Another development for such an application has resulted in a device providing a broadband plasma jet (about $30 \mathrm{~cm}$ wide). This jet is generated by the reactant gas flowing across an arc which is stabilized partially by a cascade of constrictors and partially by magnetic fields [17]. An economical advantage is offered if these processes can take place at atmospheric pressure, even if lower pressures may yield higher rates. However, dielectric barrier discharges or atmospheric-pressure glow discharges may prove to be strong competition because of lower heat generation.

Plasma synthesis of nanoparticles with diameters between 5 and $50 \mathrm{~nm}$ has seen much renewed interest with the emergence of nanophase materials with specific desirable properties. A thermal plasma process provides high reactant flow and particle generation rates. The characteristic process time can be considered the time from reactant injection to nucleation and is in the range between 0.01 to $1 \mathrm{~ms}$. While the generation of such particles consisting of a large variety of materials using a plasma process has been used for over 30 years, the problem has been to realize the advantageous properties in bulk materials made from these particles. One solution is offered by a new approach in which the particles are nucleated in proximity of the end-product. As an example, consider the Hypersonic Plasma Particle Deposition process, in which reactants are injected into the jet of a DC plasma torch and dissociated, and the plasma is then accelerated in a supersonic nozzle. The cooling of the gas mixture through expansion results in nucleation and formation of particles with a narrow size distribution peaking at about $20 \mathrm{~nm}$. The very high velocities of the particles (calculations indicate velocities of 2500 to 3000 $\mathrm{m} / \mathrm{s}$ ) allow inertial deposition of these particles on a substrate placed in front of the nozzle exit [18]. In a variation of this process, the particles are directed through a series of aerodynamic focusing lenses, resulting in features of 30 to $50 \mu \mathrm{m}$ line width consisting of nanosize particles (see Fig. 3) [19]. 


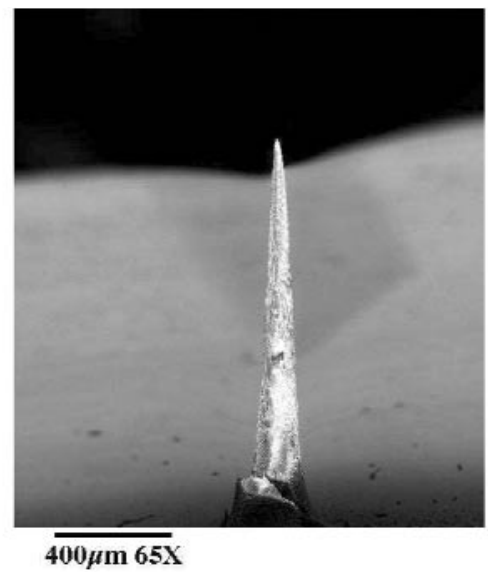

Fig. 3 Scanning electron microscope image of a microscopic feature consisting of SiC nanoparticles deposited with the HPPD process [19].

In plasma waste treatment, the major advantages of using thermal plasmas in this applications are the fast heating rates, the high processing temperatures allowing the formation of stable vitrified slags, and the low off-gas flow rates. Off-gas cleaning is a major economic factor in any waste processing installation, and the costs scale with increasing gas flows. The major issue is the economics of the specific process, and all new developments have been directed toward improving the economics either by combining plasma processes with conventional incinerators to make use of the heating value of the wastes, or by using the waste heat for obtaining a useful co-product. In situ vitrification of the incinerator ash is an example for a hybrid process, while the generation of a combustible gas is an example of generating a usable co-product. An interesting development has come out of a cooperation between Hitachi and Westinghouse Plasma Systems, where waste is treated in a plasma-fired cupola [20]. Since the processing gas (oxygen or air) is preheated by the plasma torches, lower gas flow rates are required for the waste treatment process than in normal incinerators, and the off-gas contains a high percentage of CO. In a secondary combustion chamber, steam is generated to drive a turbine. Process economics may be further improved by casting the vitrified slag into usable tiles.

\section{NEW APPROACHES IN THERMAL PLASMA MODELING AND DIAGNOSTICS}

The principal motivation for new approaches in the characterization of thermal plasmas has been the goal to describe realistic conditions, i.e., thermal plasmas including gradients and nonthermal boundaries. Increased computing power allowed the introduction of more sophisticated numerical treatments. Examples are the 3-D description of a plasma reactor [21] or the 3-D description of a plasma spray jet [22] or the nonequilibrium between electrons and heavy particles in "nonthermal" regions of an electric arc [23]. In a number of modeling approaches, the description is not confined to the thermal plasma and its boundaries, but also includes the material to be processed [e.g., 24-26].

One of the continued problems encountered in thermal plasmas is the description of mass transport by diffusion. Several new approaches have been proposed based on different formalisms [27,28]. The description of the plasma chemistry nowadays almost universally uses chemical kinetics calculations combined with the fluid dynamics description. This allows a realistic modeling characterization of the chemical nonequilibrium in the plasma boundary layers. However, only limited attempts have been made in the description of time-dependent effects such as some of the plasma instabilities, usually by imposing some arbitrary fluctuation [e.g., 29]. 
A significant effort has been spent on developing laser-based diagnostics which offer the promise of not only having high spatial and temporal resolution, but also of being independent of the assumption of local thermodynamic equilibrium. Thomson and Rayleigh scattering as well as CARS have been used for characterization of arcs and turbulent plasma jets [30-32], and results have given some information on chemical and thermal nonequilibrium. However, the assumptions made for the data reduction may pose comparable restrictions to the validity of the data as the assumption of LTE.

A different approach in obtaining relevant data on thermal plasmas has been pursued by using relatively simple diagnostics with extensive (but on-line) computer analysis, e.g., voltage waveform analysis [33] or acoustic analysis of plasma jets [11]. Frequently, these types of diagnostics are usable in process control systems.

A significant effort is being expanded in the characterization of the plasma instabilities. The efforts concentrate on identifying characteristics of experimentally determined arc power fluctuations through analysis of voltage waveforms or of fluctuations of the emitted light and sound [33-36]. Data analysis methods have included use of chaos theory to identify higher order effects [37,38]. Even more interesting are efforts to generate instabilities in a controlled manner by discharge modulation [39-41]. In particular, plasma modulations have been applied to investigate the different relaxation times for heating of the different species and for the chemical reactions.

\section{CONCLUSIONS}

The use of thermal plasmas in industry has been expanding in applications where the unique characteristics could be translated into economic advantages. The control of these processes means to achieve a fundamental understanding and control of the plasma instabilities and the conditions in the plasma boundaries. This has been achieved by the use of combinations of thermal plasmas with either other discharges (hybrid discharges) or with other processing methods, or by discharge modulation. In all these approaches, the improved control has been due to the establishment of chemical or thermal nonequilibrium. Improved control of the plasma instabilities has been obtained through fixed arc-electrode attachments or by the use of fluid dynamic shrouds. Fundamental studies have used new capabilities in modeling and simulation as well as in diagnostic techniques to investigate such nonequilibrium regions. It appears that much of the work related to thermal plasma technology concentrates on characterizing the nonthermal boundaries of the thermal plasma, and on enhancing the nonequilibrium conditions in a controlled manner.

\section{ACKNOWLEDGMENTS}

This work has been supported by NSF through Grant No. CTS-9903950, and by the Materials and Engineering Research Division of the DOE Basic Energy Science Department.

\section{REFERENCES}

1. D. Neuschütz. High Temp. Mater. Proc. 4, 127 (2000).

2. Northwest Mettech Corp. Richmond, BC/Canada. Product information bulletin on Axial III Trielectrode Plasma Torch (1998).

3. J. Zierhut, P. Haselbeck, K. D. Landes, G. Barbezat, M. Müller, M. Schutz. In Thermal Spray: Meeting the Challenges of the $21^{\text {st }}$ Century, C. Coddet (Ed.), pp. 1369-1374, ASM International, Materials Park, OH (1998).

4. H. C. Chen, Z. Duan, J. Heberlein, E. Pfender. In Thermal Spray: Practical Solutions for Engineering Problems, C. C. Berndt (Ed.), pp. 533-561, ASM International, Materials Park, OH (1996). 
5. K. Mailhot, F. Gitzhofer, M. I. Boulos. In Thermal Spray: A United Forum for Scientific and Technological Advances, C. C. Berndt (Ed.), pp. 21-25, ASM International, Materials Park, OH (1997).

6. E. Boyer, F. Gitzhofer, M. Boulos. In Thermal Spray: Practical Solutions for Engineering Problems, C. C. Berndt (Ed.), pp. 683-691, ASM International, Materials Park, OH (1996).

7. T. Yoshida, T. Tani, H. Nishimura, K. Akashi. J. Appl. Phys. 54, 640 (1983).

8. C. Moreau, P. Gougeon, M. Lamontagne, V. Lacasse, G. Vandeuil, P. Cielo. In Thermal Spray Industrial Applications, C. Berndt, S. Sampath (Eds.), pp. 431-438, ASM International, Materials Park, OH (1994).

9. W. D. Swank, J. R. Fincke, D. C. Haggard. In Thermal Spray Science and Technology, C. C. Berndt and S. Sampath (Eds.), pp. 111-116, ASM International, Materials Park, OH (1995).

10. J. Zierhut, K. D. Landes, W. Krömmer, P. Heinrich. In Thermal Spray: Surface Engineering via Applied Research, C. C. Berndt (Ed.), pp. 63-66, ASM International, Materials Park, OH (2000).

11. Z. Duan, L. Beall, J. Schein, J. Heberlein, M. Stachowicz. J. Therm. Spray Tech. 9, 225 (2000).

12. N. Ohtake and M. Yoshikawa. J. Electromechanical Soc. 137, 717 (1990).

13. R. L. Woodin, L. K. Bigelow, G. L. Cann. In Applications of Diamond Films and Related Materials, Y. Tzeng, M. Yoshikawa, M. Murakawa, A. Feldman (Eds.), pp. 439-444, ASM International, Materials Park, OH (1991).

14. S. K. Baldwin, Jr., T. G. Owano, C. H. Kruger. Appl. Phys. Lett. 67, 194 (1995).

15. O. B. Postel and J. V. R. Heberlein. Dia. Rel. Mater. 8, 1878 (1999).

16. P. Koulik, S. Begounov, S. Goloviatinskii. Plasma Chem. Plasma Proc. 19, 311 (1999).

17. R. Hartmann and K. D. Landes. In Thermal Spray: Surface Engineering via Applied Research, C. C. Berndt (Ed.), pp. 15-20, ASM International, Materials Park, OH (2000).

18. J. Blum, N. Tymiak, A. Neuman, Z. Wong, N. P. Rao, S. L. Girshick, W. W. Gerberich, P. H. McMurry, J. V. R. Heberlein. J. Nanoparticle Res. 1, 31 (1999).

19. F. Di Fonzo, A. Gidwani, M. H. Fan, D. Neumann, D. I. Iordanoglu, J. V. R. Heberlein, P. H. McMurry, S. L. Girshick, N. Tymiak, W. W. Gerberich. Appl. Phys. Lett. 77, 910 (2000).

20. Hitachi Metals Corp. Product bulletin 2000-11. <www.hitachi-metals.co.jp $>$ or $<$ www.westinghouse-plasma.com>.

21. C. Delalondre, M. Gonzalez, O. Simonin, S. Zahrai. High Temp. Mater. Proc. 2, 129 (1998).

22. G. Mariaux, C. Bandry, A. M. Vardelle. In Thermal Spray 2001: New Surfaces for a New Millenium, C. C. Berndt, K. A. Khor, E. F. Lugscheider (Eds.), pp. 933-942, ASM International, Materials Park, OH (2001).

23. J. Haider. J. Phys. D: Appl. Phys. 32, 263 (1999).

24. B. Ravary, L. Fulcheri, G. Flamant, J. Bakken. Plasma Chem. Plasma Proc. 19, 69 (1999).

25. P. Fauchais, A. Vardelle, M. Vardelle, A. Denoivjean, B. Pateyron, M. El Ganaoui. In Thermal Spray 2001: New Surfaces for a New Millenium, C. C. Berndt, K. A. Khor, E. F. Lugscheider (Eds.), pp. 865-873, ASM International, Materials Park, OH (2001).

26. M. Pasandideh-Fard and J. Mostaghimi. In Thermal Spray: Surface Engineering via Applied Research, C. C. Berndt (Ed.), pp. 125-134, ASM International, Materials Park, OH (2000)

27. J. D. Ramshaw and C. H. Chang. J. Non-Equilibrium Thermodynamics 21, 223 (1996).

28. A. B. Murphy. High Temp. Mater. Proc. 4, 1 (2000).

29. J. H. Park, J. Heberlein, E. Pfender, Y. C. Lau, J. Ruud, H. P. Wang. In Proc. $2^{\text {nd }}$ International Symp. Heat and Mass Transfer Under Plasma Conditions, P. Fauchais, J. van der Mullen, J. Heberlein (Eds.), pp. 417-424, New York Academy of Sciences (1999).

30. S. C. Snyder, L. D. Reynolds, J. R. Fincke, G. D. Lassahn, J. D. Grandy, T. E. Repetti. Phys. Rev. E. 50, 519 (1994).

31. R. E. Bentley. J. Phys. D. 30, 2880 (1997).

32. G. Gregori, J. Schein, P. Schwendinger, U. Kortshagen, J. Heberlein, E. Pfender. Phys. Rev. E. 59, 2286 (1999). 
33. Z. Duan and J. Heberlein. In Thermal Spray: Surface Engineering via Applied Research, C. C. Berndt (Ed.), pp. 1-7, ASM International, Materials Park, OH (2000).

34. J. F. Coudert and P. Fauchais. High Temp. Mater. Proc. 1, 149 (1997).

35. B. G. Chevon, A. Bultel, B. Mahen. High Temp. Mater. Proc. 2, 69 (1998).

36. A. Kaminska and M. Dudeck. High Temp. Mater. Proc. 2, 117 (1998).

37. G. Bonhomme, A. Atipo, X. Caron, E. Gravier. High Temp. Mater. Proc. 2, 103 (1998).

38. J. Sheard, J. Heberlein, K. Stelson, E. Pfender. In Thermal Spray: A United Forum for Scientific and Technological Advances, C. C. Berndt (Ed.), pp. 613-618, ASM International, Materials Park, OH (1997).

39. V. Lago, A. Lebehot, M. Dudlek. High Temp. Mater. Proc. 3, 385 (1999).

40. T. Sakuta. In Proc. $14^{\text {th }}$ Int'l. Symp. Plasma Chem., M. Hrabovsky, M. Konrad, V. Kopecky (Eds.), pp. 14-16, Inst. Plasma Czech Academy of Sciences, Prague, Czech Republic (1999).

41. B. Goodman. In Thermal Spray Industrial Applications, C. C. Berndt and S. Sampath (Eds.), pp. 367-370, ASM International, Materials Park, OH (1994). 